\title{
Proteomic Analysis of Protein Posttranslational Modifications by Mass Spectrometry
}

\author{
Danielle L. Swaney and Judit Villén ${ }^{1}$ \\ Department of Genome Sciences, University of Washington, Seattle, Washington 98195
}

\begin{abstract}
The addition of posttranslational modifications (PTMs) to proteins is an influential mechanism to temporally control protein function and ultimately regulate entire cellular processes. Most PTMs are present at low stoichiometry and abundance, which limits their detection when analyzing whole cell lysates. PTM purification methods are thus required to comprehensively characterize the presence and dynamics of PTMs using mass spectrometry-based proteomics approaches. Here we describe several of the most influential PTMs and discuss the fundamentals of proteomics experiments and PTM purification methods.
\end{abstract}

Protein posttranslational modifications (PTMs) modulate protein functions and are integral to almost every cellular process. The reversible nature of PTMs enables dynamic regulation of PTM statusfunctioning as highly versatile switches to control protein structure, activity, subcellular localization, etc. PTMs can function individually or can work in a coordinated fashion with other PTMs. For example, multiple PTMs on the same protein can interact to alter protein function, or individual PTMs on different proteins can interact in series to form interconnected signaling networks and regulate broad cellular processes. To modulate the PTM status of proteins, specific classes of enzymes catalyze the addition of particular modification types to proteins (e.g., kinases and acetyl transferases), whereas alternate classes of enzymes are responsible for the removal of PTMs (e.g., phosphatases and deacetylases). More than 200 different types of PTMs have been described; however, most exist at low stoichiometry on proteins of low abundance (Krishna and Wold 1998; Mann and Jensen 2003). Therefore, to characterize PTMs on a proteome-wide scale, purification methods are necessary to isolate modified proteins and peptides before proteomic analysis. In the associated protocols (Protocol: Enrichment of Phosphopeptides via Immobilized Metal Affinity Chromatography [Swaney and Villén 2015a] and Protocol: Enrichment of Modified Peptides via Immunoaffinity Precipitation with Modification-Specific Antibodies [Swaney and Villén 2015b]), we describe such purification methods for the proteomic analysis of several of the most widely studied PTMs.

\section{TYPES OF POSTTRANSLATIONAL MODIFICATIONS}

Arguably the most well-studied PTM is protein phosphorylation, for which more than 20,000 phosphorylation sites have been detected in yeast, targeting nearly half of the yeast proteome (Stark et al.

\footnotetext{
${ }^{1}$ Correspondence: jvillen@u.washington.edu

(C) 2016 Cold Spring Harbor Laboratory Press

Cite this introduction as Cold Spring Harb Protoc; doi:10.1101/pdb.top077743
} 
2010). Kinases catalyze the transfer of a phosphoryl group from ATP to a serine, threonine, or tyrosine residue on a protein substrate. The presence of phosphorylation on a specific amino acid can be detected in mass spectrometry-based proteomics experiments by a characteristic mass addition of 79.96633 Da. Phosphorylation is a reversible PTM and can be removed from a substrate by phosphatase enzymes. This reversible nature enables proteins to be dynamically modified to meet cellular needs. For example, cell cycle progression requires a network of phosphorylation events that temporally oscillate to activate protein substrates. Also, protein phosphorylation is the main driving force behind signal transduction.

Another widely studied PTM is ubiquitylation. Ubiquitin is a small $(8.6-\mathrm{kDa})$ protein that is added to substrates via a multistep enzymatic cascade and can be removed by a class of proteins known as deubiquitinating enzymes (DUBs). To add ubiquitin to a substrate, ubiquitin is first activated by an enzyme (E1) and conjugated to a second enzyme (E2). In most cases, a ligase enzyme (E3) recognizes a substrate and complexes with it and the E2 to facilitate transfer of ubiquitin to a lysine residue on the substrate. Unlike phosphorylation and many other PTMs that are monomeric, ubiquitin can be added to a substrate in a polymeric fashion. Here, ubiquitin can serve as both modification and substrate to generate a branched structure propagated through one of its seven lysine residues. This branching adds additional layers of complexity to ubiquitin signaling. Different branching structures have been associated with specific cellular processes. The most abundant branching structure through lysine 48 on ubiquitin (K48) is commonly used to mark proteins for degradation, whereas branching through lysine 63 (K63) is associated with the DNA damage response, signaling, and trafficking of membrane proteins (Spence et al. 1995). Because of the size of the ubiquitin, and its capacity for polymeric addition, proteomics analysis relies on indirect detection methods. Enzymatic proteolysis of lysates with trypsin leaves the two carboxy-terminal glycine residues of ubiquitin attached to lysine. Antibodies with specificity to this diglycyl-lysine residue can then be used to purify sites of lysine ubiquitylation (Emanuele et al. 2011; Kim et al. 2011; Wagner et al. 2011), and detection of a mass addition of 114.04293 Da on lysine residues can confirm the site of ubiquitylation (Peng and Gygi 2001). A caveat of this approach is that other modifications by ubiquitin-like proteins can also produce a diglycyl-lysine residue (e.g., neddylation). In yeast, antibody-based enrichment of diglycyl-lysine has identified more than 5500 ubiquitylation sites on $\sim 1900$ proteins, revealing that at least onethird of the proteome is ubiquitylated (Swaney et al. 2013).

The final PTM that has been well characterized in yeast is lysine acetylation. Here, lysine acetyltransferases (KATs; also known as histone acetyltransferases, HATs) catalyze the addition of an acetyl group onto lysine residues. This modification is also reversible and can be removed by lysine deacetylases (KDACs; also known as histone deacetylases, HDACs). Using mass spectrometry detection, the characteristic mass addition of $42.01056 \mathrm{Da}$ on lysine can confirm the presence of acetylation. Proteomic experiments using immunoaffinity purification methods have revealed $\sim 4000$ sites of lysine acetylation in yeast (Henriksen et al. 2012). This profiling has helped illustrate roles for lysine acetylation signaling in yeast, such as mitochondrial metabolism, protein synthesis, and chromatin organization (Henriksen et al. 2012).

\section{PROTEOMIC METHODS}

Mass spectrometry-based proteomics is a powerful approach to identify proteins modified by a particular PTM. Additionally, characteristic mass shifts in fragmentation spectra (i.e., tandem mass spectrometry [MS/MS]) often permit exact localization of the PTM to an individual amino acid within the protein. Advances in both methodology and technology have dramatically propelled the field of PTM analysis, and over the past decade, the number of PTM sites identified in a proteomics experiment has increased more than 30-fold (Ficarro et al. 2002; Peng et al. 2003; Swaney et al. 2013). In a single proteomics experiment, researchers can now routinely identify thousands of PTMs, as well as quantify their abundance changes in response to stimuli (Olsen et al. 2006; Kim et al. 2011; Henriksen et al. 2012). Such advancements have illustrated the breadth and diversity of modified proteins that regulate cell state, although the challenge of defining the biological function of these PTMs still remains. 
Mass spectrometry experiments to characterize PTMs are often separated into single protein or global proteomics approaches. In single protein experiments, mass spectrometry is performed on peptides following isolation of a given protein of interest. This approach is useful for comprehensive identification of the variety of PTMs on a single protein; however, the success of this approach is dependent on effective isolation of the protein of interest. Two common strategies are protein-level immunoprecipitation using antibodies and introduction of a tag to the protein that can be used for purification (e.g., GST, FLAG, His-tag). Such isolation can be highly protein-specific, and thus methods for this approach are not described here.

Alternatively, using a global proteomics approach, one can obtain a cellular wide view of the PTM status of thousands of proteins at once. Here, peptides containing a single PTM type (e.g., phosphorylation) are isolated from a complex mixture of thousands of peptides. Methods to isolate modified peptides largely fall into two categories. First are biochemical-based methods, which exploit differences in chemical properties between modified peptides and nonmodified peptides (e.g., solution charge, metal affinity). This approach is used to isolate phosphopeptides by immobilized metal affinity chromatography (IMAC); see Protocol: Enrichment of Phosphopeptides via Immobilized Metal Affinity Chromatography (Swaney and Villén 2015a). The second category consists of immunoaffinity enrichment methods in which antibodies with specificity toward a particular PTM of interest (e.g., acetylation, ubiquitylation, phosphotyrosine) are used to purify PTM containing peptides; see Protocol: Enrichment of Modified Peptides via Immunoaffinity Precipitation with ModificationSpecific Antibodies (Swaney and Villén 2015b).

Phosphorylation, ubiquitylation, and acetylation represent the PTMs that are most widely studied using global proteomics methods. As mentioned, many additional PTMs exist and regulate important biological processes. However, the study of these PTMs remains a challenge, as sensitive and selective purification methods for most of them have yet to be described.

\section{ACKNOWLEDGMENTS}

This work was supported in part by National Institutes of Health/National Cancer Institute (NIH/ NCI) grant R00CA140789 and an Ellison Medical Foundation New Scholar Award (to J.V.).

\section{REFERENCES}

Emanuele MJ, Elia AEH, Xu Q, Thoma CR, Izhar L, Leng Y, Guo A, Chen Y-N, Rush J, Hsu PW-C, et al. 2011. Global identification of modular cullin-RING ligase substrates. Cell 147: 459-474.

Ficarro SB, McCleland ML, Stukenberg PT, Burke DJ, Ross MM, Shabanowitz J, Hunt DF, White FM. 2002. Phosphoproteome analysis by mass spectrometry and its application to Saccharomyces cerevisiae. Nat Biotechnol 20: 301-305.

Henriksen P, Wagner SA, Weinert BT, Sharma S, Bacinskaja G, Rehman M, Juffer AH, Walther TC, Lisby M, Choudhary C. 2012. Proteome-wide analysis of lysine acetylation suggests its broad regulatory scope in Saccharomyces cerevisiae. Mol Cell Proteomics 11: 1510-1522.

Kim W, Bennett EJ, Huttlin EL, Guo A, Li J, Possemato A, Sowa ME, Rad R, Rush J, Comb MJ, et al. 2011. Systematic and quantitative assessment of the ubiquitin-modified proteome. Mol Cell 44: 325-340.

Krishna RG, Wold F. 1998. Posttranslational modifications. In Proteins: Analysis and design (ed. Angeletti RH), pp. 121-207. Academic Press, San Diego.

Mann M, Jensen ON. 2003. Proteomic analysis of post-translational modifications. Nat Biotechnol. 21: 255-261.

Olsen JV, Blagoev B, Gnad F, Macek B, Kumar C, Mortensen P, Mann M. 2006. Global, in vivo, and site-specific phosphorylation dynamics in signaling networks. Cell 127: 635-648.

Peng J, Gygi SP. 2001. Proteomics: The move to mixtures. J Mass Spectrom 36: 1083-1091.
Peng J, Schwartz D, Elias JE, Thoreen CC, Cheng D, Marsischky G, Roelofs J, Finley D, Gygi SP. 2003. A proteomics approach to understanding protein ubiquitination. Nat Biotechnol 21: 921-926.

Spence J, Sadis S, Haas AL, Finley D. 1995. A ubiquitin mutant with specific defects in DNA repair and multiubiquitination. Mol Cell Biol 15: $1265-1273$.

Stark C, Su T-C, Breitkreutz A, Lourenco P, Dahabieh M, Breitkreutz B-J, Tyers M, Sadowski I. 2010. PhosphoGRID: A database of experimentally verified in vivo protein phosphorylation sites from the budding yeast Saccharomyces cerevisiae. Database (Oxford) 2010: bap026.

Swaney DL, Villén J. 2015a. Enrichment of phosphopeptides via immobilized metal affinity chromatography. Cold Spring Harb Protoc doi: 10.1101/pdb.prot088005.

Swaney DL, Villén J. 2015b. Enrichment of modified peptides via immunoaffinity precipitation with modification-specific antibodies. Cold Spring Harb Protoc doi: 10.1101/pdb.prot088013.

Swaney DL, Beltrao P, Starita L, Guo A, Rush J, Fields S, Krogan NJ, Villén J. 2013. Global analysis of phosphorylation and ubiquitylation cross-talk in protein degradation. Nat Methods 10: 676-682.

Wagner SA, Beli P, Weinert BT, Nielsen ML, Cox J, Mann M, Choudhary C. 2011. A proteome-wide, quantitative survey of in vivo ubiquitylation sites reveals widespread regulatory roles. Mol Cell Proteomics 10: M111.013284. 


\section{Proteomic Analysis of Protein Posttranslational Modifications by Mass Spectrometry}

Danielle L. Swaney and Judit Villén

Cold Spring Harb Protoc; doi: 10.1101/pdb.top077743

\begin{tabular}{|c|c|}
\hline $\begin{array}{l}\text { Email Alerting } \\
\text { Service }\end{array}$ & Receive free email alerts when new articles cite this article - click here. \\
\hline \multirow{2}{*}{$\begin{array}{l}\text { Subject } \\
\text { Categories }\end{array}$} & Browse articles on similar topics from Cold Spring Harbor Protocols. \\
\hline & $\begin{array}{l}\text { Affinity and Immunoaffinity Chromatography (57 articles) } \\
\text { Characterization of Proteins (208 articles) } \\
\text { Chromatography (47 articles) } \\
\text { Mass Spectrometry (78 articles) } \\
\text { Phosphorylation Site Mapping (16 articles) } \\
\text { Protein Identification and Analysis (202 articles) } \\
\text { Proteins and Proteomics, general (575 articles) } \\
\text { Proteomics (66 articles) }\end{array}$ \\
\hline
\end{tabular}

